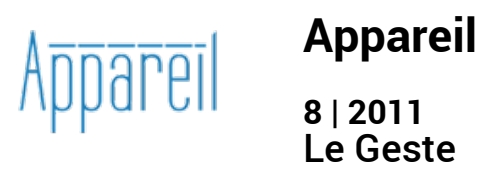

\title{
Pour un geste du préalable, lorsque l'intention se fait forme
}

Arts Plastiques et chorégraphie

Mélanie Perrier

\section{OpenEdition}

\section{Journals}

Édition électronique

URL : http://journals.openedition.org/appareil/1291

DOI : 10.4000/appareil. 1291

ISSN : 2101-0714

Éditeur

MSH Paris Nord

Référence électronique

Mélanie Perrier, «Pour un geste du préalable, lorsque l'intention se fait forme », Appareil [En ligne], 8 |

2011, mis en ligne le 26 octobre 2011, consulté le 30 juillet 2020. URL : http://

journals.openedition.org/appareil/1291 ; DOI : https://doi.org/10.4000/appareil.1291

Ce document a été généré automatiquement le 30 juillet 2020.

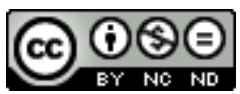

Appareil est mis à disposition selon les termes de la Licence Creative Commons Attribution - Pas d'Utilisation Commerciale - Pas de Modification 4.0 International. 


\title{
Pour un geste du préalable, lorsque l'intention se fait forme
}

\author{
Arts Plastiques et chorégraphie
}

Mélanie Perrier

1 Jusqu'à présent, un schéma dominait la perception du processus de création artistique : il admettait le passage de l'intention à la réalisation de manière linéaire : l'artiste vérifiait et reconnaissait ses intentions dans les effets de ces actes.

2 Le $\mathrm{xx}^{\mathrm{e}}$ siècle a mis à mal cette conception de l'art comme mise en forme d'une pensée symbolique, en revendiquant le hasard, l'automatisme, (des surréalistes à John Cage) en passant par l'invention de techniques non conventionnelles. Les gestes ont pris ainsi une importance singulière, participant à la désincarnation et à la distance avec la matière au profit du concept.

3 Nous sommes alors passés "d'une foi en la technologie à une foi des gestes ${ }^{1}$ ", où s'exposent désormais les processus, les systèmes de création. Et les objets finis et récupérables disparaissent progressivement au détriment d'un agir, d'un concept ou d'une intention. Avec pareille démarche, c'est la relation historique entre penser et faire qui est mise à mal, instaurant désormais un primat de l'idée sur sa formalisation.

\section{Gestes}

4 Pour sortir de cette dichotomie penser / faire, le geste peut paradoxalement se constituer comme un outil opérant pour envisager certaines pratiques artistiques contemporaines, précisément parce qu'il a su migrer au fil des 60 dernières années, du domaine $\mathrm{du}$ faire au domaine $\mathrm{du}$ penser, en se délestant de l'incarnation de la technique.

5 Le geste, comme un mouvement du corps caractériserait non plus un acte de création artistique mais plutôt une forme d'agir. Le geste passerait ainsi du statut d'une mise en forme de la pensée à la visibilité d'une intention. 
6 Ce déplacement de la place du geste, redéfinit par voie de conséquence la relation qu'il entretenait jusqu'alors avec l'intention initiale de l'artiste ainsi que ses modes de visibilité. Qu'est ce qui fait encore intention dans une œuvre? Quels en sont ses contours par rapport au geste?

7 L'intention est tantôt considérée comme visée de l'œuvre immanente à l'artiste-auteur, tantôt relevant de l'héritage Duchampien pour qui elle devient objet même du travail artistique.

8 Dans le premier cas, l'intention augure une consistance (qui ne se résout pas à la simple énonciation de l'intention) et réclame un acte d'instauration sollicitant les savoir-faire de l'artiste. L'intention s'impose ici comme l'amorce d'une manifestation, celle d'un projet à finaliser en objet ou en événement, l'œuvre.

9 Dans le deuxième cas, l'intention s'émancipe de sa manifestation : elle ne serait plus le schéma d'exécution mais se prétendrait acte même d'œuvre. Un des grands bouleversements opérés dans la seconde moitié $\mathrm{du} \mathrm{xx}^{\mathrm{e}}$ siècle.

On ne peut dès lors nier les échos Hegeliens qui affleurent, si on rapproche l'intention de l'idée. En effet, que proposait Hegel si ce n'est l'idée comme principe de déploiement de l'art dans des formes artistiques. Ainsi dit-il :

Les formes artistiques ne sont rien d'autre que les différents rapports qu'entretiennent le contenu et la figure, rapports qui procèdent de l'Idée elle-même et fournissent de ce fait le véritable principe de division, de répartition, de cette sphère ${ }^{2}$.

11 Il s'agit bien pour Hegel d'expliciter la vérité de l'art dans sa capacité à rendre effectif un contenu spirituel, et non d'accorder une importance au contenu.

12 Hegel nous amène donc à réévaluer la relation entre l'intention et la forme qu'elle prend, pour peut-être même s'extraire d'une simple causalité.

13 L'intention s'apparente ainsi davantage à une volonté de l'artiste: celle d'un projet d'œuvre, inaugurant et portant en lui-même un trajet à venir.

Car « l'intention n'est pas le projet d'un butin mais bien la vertu d'un chemin ${ }^{3}$. » précise Georges Amar. L'artiste est dès lors à l'origine de son œuvre sans être son point de capitonnage. Il est l'intention de l'œuvre, celle qui délimite un commencement et non un prochain déroulement.

15 «L'intention n'est pas une décision, arbitraire et calculée, mais la naissance d'une relation » poursuit Georges Amar. Dessiner les traits d'un préalable plus qu'assurer ceux d'un aboutissement. Tels seraient ainsi les contours de l'intention dans certaines démarches artistiques d'aujourd'hui.

Autrement dit, l'intervention de l'artiste semble délaisser le strict terrain du faire et trouver sa légitimité dans la prédominance de l'intention : celle d'un projet d'œuvre qui n'oublie pas pour autant son effectivité. L'artiste devient celui qui projette, initie, inaugure, débute, ouvre, invite, accueille, occasionne.

Quoiqu'il en soit, il serait insuffisant d'identifier l'artiste à un simple " concepteur ${ }^{4}$ ». Car même si le projet prime sur le trajet, même si la persistance n'est plus celle du matériau mais "cognitive» dira Paul Virilio ${ }^{5}$, il appartient à l'artiste/producteur d'intention de proposer également les stratégies d'effectivité de son intention.

18 Aussi gardons pour l'heure l'intention non plus comme expression de l'esprit de l'artiste mais comme induction de création. 
Citons comme première borne à cette hypothèse, la célèbre Declaration of Intent (déclaration d'intention) de l'artiste Lawrence Weiner qu'il formula en 1968 :

1. L'artiste peut concevoir l'œuvre. 2. L'œuvre peut être fabriquée. 3. L'œuvre n'a pas besoin d'être faite. Chaque partie étant de même valeur et en cohérence avec l'intention de l'artiste, la décision comme la situation repose pour le récepteur sur les modalités de la règle.

Ses œuvres, qu'il nomme sculptures, se présentent alors sous la forme d'énoncés qui désignent des objets et des actions se construisant dans l'esprit du spectateur.

Geste radical propre à l'Art conceptuel en plein essor à l'époque, cette déclaration impose désormais l'énonciation comme geste fondateur et fondamental de l'œuvre. Énoncer c'est faire.

L'existence de l'œuvre ne dépend plus de sa traduction dans le réel, elle peut éternellement rester sous forme d'idée ou bien écrite, archivée dans un tiroir que personne n'ouvrira. C'est ce qu'on appelle aujourd'hui « les protocoles artistiques ».

Ces œuvres protocolaires interrogent alors en amont de leur déroulement les limites de leur mode d'existence. "Auto réflexifs", ces gestes énonciateurs et préalables nous conduisent à nous interroger sur les modes d'apparition et d'apparence de l'art.

Les manières dont ces gestes d'énonciation se donnent à voir passent d'abord par des graphies prescriptives : des partitions, fascicules, mode d'emploi, indications, et autres statements dévoilés et exposés par les artistes. Aussi qui mieux que la partition relève de ce système prescriptif ? La partition n'est-elle pas initialement la transcription écrite des caractéristiques du son musical, permettant à des interprètes de reproduire la musique?

Échappée du champ musical, la partition s'est imposée dès les années 1960 tant pour les plasticiens que pour les chorégraphes comme outil élargi de création. En effet, la partition s'est pensée sur ces terrains comme processus de création et non comme notation de l'existant, ou à suivre.

Elle a très rapidement induit la question de l'interprétation de la performance ouvrant sur l'élaboration d'une partition comme système de "pré-écriture ", à l'image des happenings d'Allan Kaprow, des events de George Brecht, des statements de Weiner, autant de formes prescriptives des débuts de la performance.

27 Les chorégraphes des années 1960 délaissent alors la forme spectaculaire au profit de l'élaboration de nouvelles organisations compositionnelles pour la danse.

28 Se distinguant de l'idée de système, entité close et délimitée, mettant en œuvre une méthode spécifique, la partition se rapporte quant à elle davantage à un ou des processus. Elle les décrit, les amorce ou les dynamise. Elle inclut et induit de fait le hasard, englobe des états affectifs et composantes irrationnelles. Elle a une dimension exploratoire et non délimitée. Elle encourage le plus haut degré de créativité de la part de tous les participants, puisque l'exécution en tant qu'acte créateur résulte de la partition. Alors que le système organise, la partition guide.

29 La partition indique alors les éléments dont le rassemblement déterminera la chorégraphie ou l'œuvre qui se fera sur un mode instantané. Elle devient un dispositif ouvert susceptible de mettre en mouvement ou de créer des formes. Elle s'impose comme une matrice d'exécutions en latence, plaçant le mouvement des danseurs non plus comme une forme répétée et reproduite, mais comme les variations d'interactions organisées. 
30 L'outil partition est même, pour certains, rendu disponible ou visible pendant la performance ou la pièce. Selon Lisa Nelson, un tel « partage » démocratique des outils permet au public une observation plus fine. «... ça donne parfois des résultats très drôles quand on fait apparaître le système. "

31 Elle peut aussi se constituer comme forme autonome, à part entière. La performance n'étant qu'un moment d'activation. La partition comme dispositif d'écriture s'est ainsi autonomisée, en venant avant et non plus après la danse. Elle permet alors d'ordonner, d'organiser, de mettre ensemble, et non de prévoir ou de réglementer.

32 Si la partition comme dispositif de mise en mouvement ne donne pas d'indications formelles aux danseurs, elle intègre par contre des contraintes qui, à elles seules, délimiteront la qualité des mouvements réalisés par la suite.

33 Avec George Brecht, et ses Events, l'événement s'imagine comme « une sélection dans un univers limité de résultats possibles», produit par n'importe quelle activité et donnant lieu à un système de notation ("event score") qui le rende transmissible par ou pour n'importe qui, de façon à réduire, selon Brecht, « la distance entre hasard et choix $»$.

34 L'œuvre n'existe pas d'une manière unique et définitive, mais existe par réactivation. Elle s'expose quand elle se met en acte. Ainsi donc la partition comme geste énonciateur délimite plusieurs possibilités :

- le geste vaut pour lui-même et ne suppose pas de parachèvement matériel ou visible (Levé, Weiner) ;

- le geste prévaut et augure une réalisation ouverte (Brecht, Lehmenn) ;

- le geste annonce et ouvre sur un multiple de possibilités de réalisation (Kaprow, Thomasset, Brüggeman, IKHÉA@SERVICES) ;

- le geste délimite un mode d'emploi d'activation de l'œuvre à faire par le public (Forti, Nelson, L. Halprin)

Prenons ainsi plusieurs exemples.

Évoquons pour commencer le projet Tool Box ${ }^{6}$, initié par Jacques Rivet et Marie-Laure Viale pour Entre deux à Nantes. Cette Tool Box présente 82 « œuvres-outils » que le visiteur peut activer dans les lieux publics selon des temporalités et modalités différentes. 53 artistes ont participé en proposant chacun sur une feuille au format A4, la description textuelle d'une (ou plusieurs) œuvre(s). Ces « œuvres» se traduisent le plus souvent sous la forme de contrats, de protocoles, description ou marche à suivre déterminant les conditions d'apparition de l'œuvre. Plusieurs dénominateurs communs rassemblent ces énoncés d'œuvres :

La ponctualité et la possibilité de réactivation dans un nouveau contexte.

Une conception pour les lieux publics.

37 L'origine de leur conception remonte pour certaines aux années 1960 (Sol Lewitt, Lawrence Weiner, Vito Acconci) et couvre la période contemporaine jusqu'à nos jours. Ces œuvres prennent en compte le spectateur dans un rapport actif : soit à travers une participation directe, soit par la nature du questionnement que l'œuvre suscite chez lui.

38 En proposant des œuvres ponctuelles (éphémères et renouvelables) à réaliser, à répéter, à disséminer, à infiltrer dans les lieux publics, Tool Box réaffirme le primat de l'idée sur sa formalisation où le retrait de la figure du créateur et la délégation de la réalisation, induisent de nouveaux modes d'échanges avec le public. 
Cette dématérialisation de l'œuvre fait écho aux recherches menées par les tenants de l'art minimal: exploration du langage, réflexion sur les spécificités formelles des différents modes d'expression (sculpture, peinture, etc.), formes élémentaires. Une quête de l'essentiel qui ne confine pas pour autant à l'austérité. Ces démarches expérimentales et principes énoncés par les artistes se teintent le plus souvent d'humour, de distance critique et d'un certain esprit de subversion.

Dans un registre similaire, Stefan Brüggemann conçoit dès 2000, Show title, une liste de titres potentiels d'expositions, les shows titles, inépuisable car régulièrement complétés par l'artiste et mise à disposition librement sur son site Internet ${ }^{7}$. Les titres formulés par l'artiste déclinent des énoncés au sens suffisamment ouvert pour pouvoir prêter leur autorité nominative à un large panel de propositions.

4 Sur ce registre des possibles disponibles, citons Édouard Levé et son livre intitulé Euvres, «Un livre qui décrit des œuvres dont l'auteur a eu l'idée mais qu'il n'a pas réalisées ». Première des 533 œuvres imaginaires énumérées par l'artiste en 2002, ce livre de deux cents pages se présente comme une sorte de catalogue d'une exposition fictive. Accumulation de projets, d'idées d'œuvres, elles recouvrent tous les domaines de l'art contemporain, de la littérature aux arts plastiques, à la vidéo et la photo, en passant par les installations les plus diverses.

2 Les énoncés sont réduits à une neutralité descriptive : «Les résidus de gommage des dessins de tous les élèves d'une école des Beaux-Arts sont recueillis pendant un an et agglomérés en cube » $\left(n^{\circ} 72\right) ;$ «Un manteau en vers luisants » ( $\left.n^{\circ} 64\right)$.

De ces écritures d'œuvres ou expositions potentielles, sans visibilité nécessaire, la partition se mue également en processus de création à part entière, outil d'une création qui se dit avant de se faire.

C'est le cas de Thomas Lehmenn, chorégraphe allemand qui a créé en 2004, Funktionen Tool $^{8}$ Box, un système chorégraphique de règles et mode d'emploi de 9 pages. Ce jeu de cartes contenant plusieurs systèmes chorégraphiques permet de disposer en grand nombre de tâches, de systèmes ou juste d'inspirer ceux qui s'en saisissent. Dans ce cas, «système » doit être entendu comme une structure qui est créée mais constamment modifiée en fonction des paramètres introduits par les participants. 
Figure 1

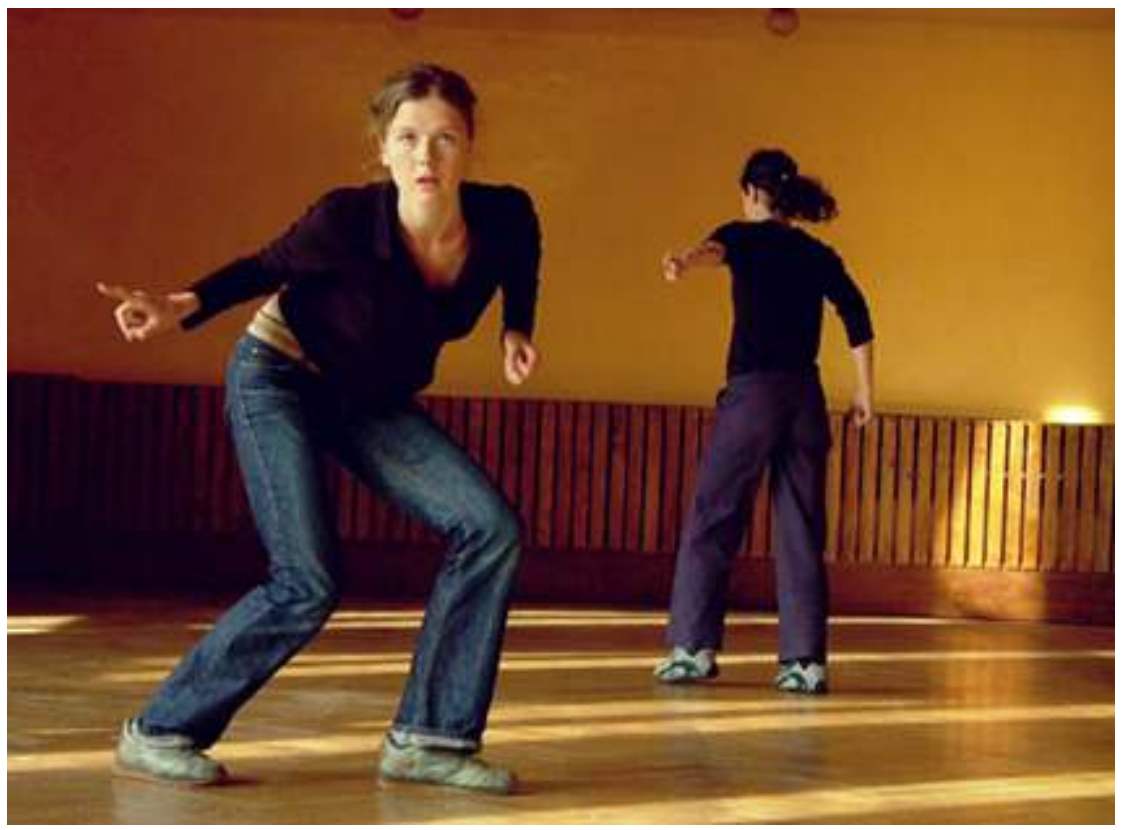

Thomas Lehmenn, FUNKTIONEN (2004)

(C) CRÉDIT PHOTO KATRIN SCHOOF

Prévue soit pour 4, 5, 7 danseurs soit pour un groupe plus large, cette Tool box contient 3 systèmes chorégraphiques différents :

- «Catégories »

- Espace (architecture, mesure, extérieur)

- Relation (entre les danseurs)

- Mouvement (mouvement physique)

- «C'est meilleur de... »

- «Fonctions»

Accompagné d'autres catégories :

- Aspects

- Thèmes

- Termes

- Personnes

- Blanc

Chaque carte peut être mise en relation avec d'autres. Dans ce sens, ces systèmes peuvent être utilisés comme décrivant en détail, pouvant changer, ou pouvant être combinés ou augmentés avec l'un l'autre. En outre, les indications initiales peuvent être améliorées avec les propres idées des participants, ou développées dans un autre système de travail au point de devenir méconnaissables dans la relation avec ce jeu initial.

La catégorie «thème » par exemple permet d'établir un lien avec les contextes sociaux particuliers afin de jeter un pont entre le monde de l'art et le monde extérieur. Si bien que la tool box peut s'utiliser également dans des cadres élargis à l'art.

49 Véritable règle $\mathrm{du}$ jeu, Tool box funktionnenn délimite un arbitraire aléatoire de combinaisons formelles. Considéré comme un groupe de structures interactives 
variables basées sur les relations entre ses composants, ce qui se donne à voir est moins le résultat formel du processus que l'activation de ce dernier.

50 La revue Véhicule se place quant à elle comme une forme d'exposition écrite de partitions d'œuvres, qui pour la plupart reste à faire.

Questionnant directement ce que peut être une partition, Véhicule, propose à chaque artiste d'en donner sa propre définition. Ainsi au gré de ses 2 numéros, la partition s'imagine en transcriptions ou notation d'une œuvre (non musicale) par son auteur même permettant à d'autres de l'interpréter, la jouer, la réaliser. Ces "partitions" ressemblent tour à tour à un kit, une instruction, une méthode, une proposition, un concept, un projet, un mode d'emploi, un déroulé, une incitation, une recette, une série d'outils, un manuel, un jeu, un ensemble permettant la construction ou la reconstitution.

Figure 2

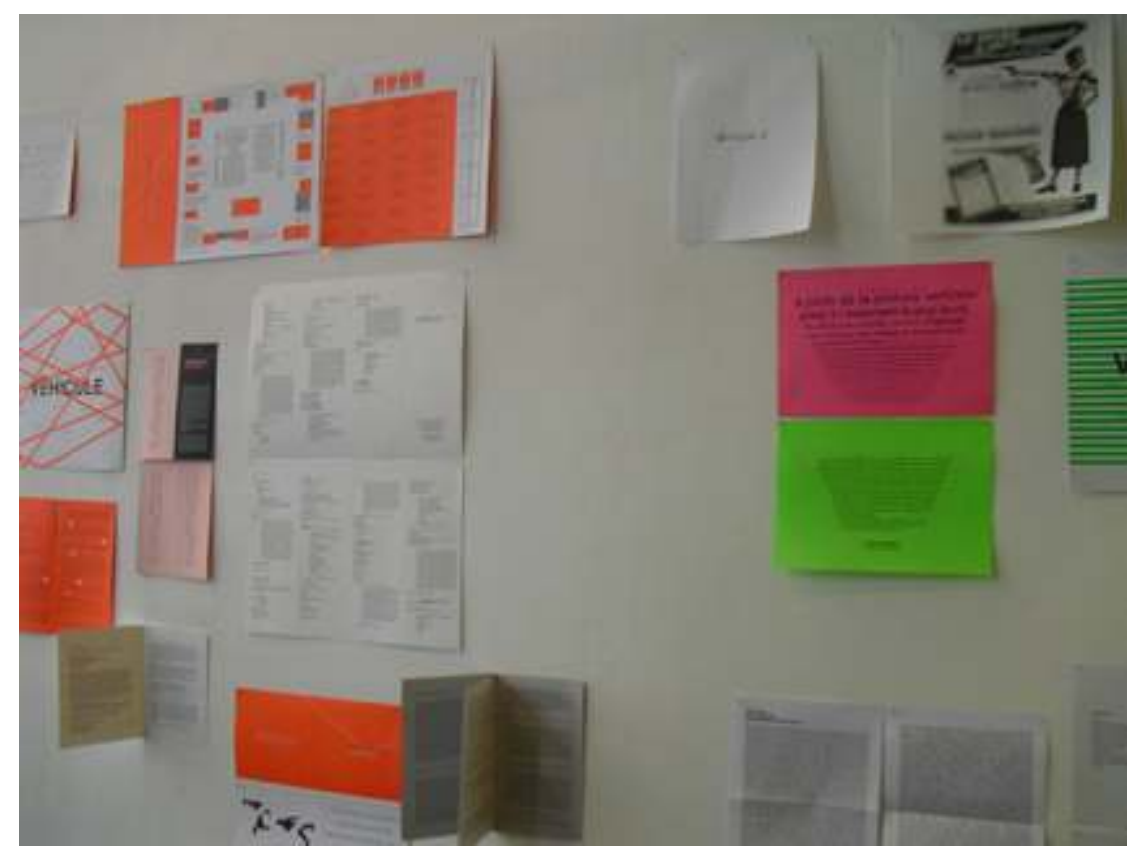

Revue véhicule exposée

(c) Crédit photo Mélanie Perrier

Vincent Thomasset, dans Véhicule 1 délimite quant à lui Plugs 9 un mode d'emploi d'une pratique, susceptible d'être activée seul ou à plusieurs (public ou danseurs) : auto plug, blitz plug, slow plug, 4 bras, 4 jambes, total plug, etc. Ce protocole d'action consiste à essayer, dans la mesure du possible, à introduire bras, mains, jambes, pieds pour se retrouver bloqué. Une fois immobilisés, les pluggers s'arrêtent un temps, peuvent essayer de se déplacer, puis se dépluggent membre par membre ${ }^{10}$. 
Figure 3

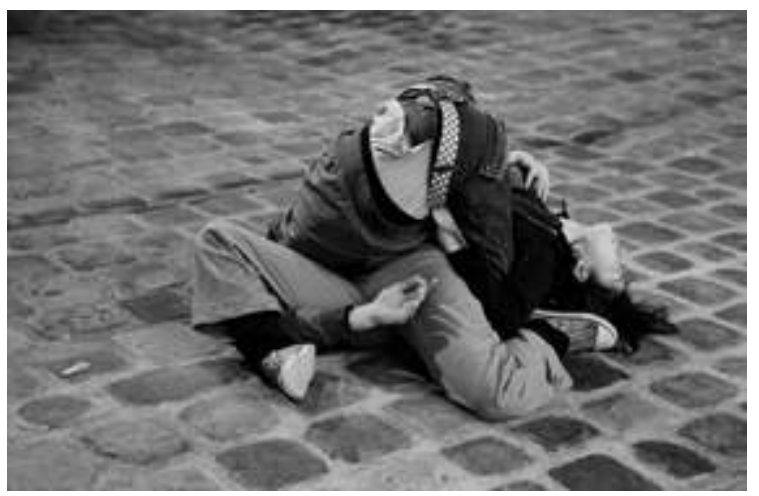

Vincent Thomasset, Plugs (2009)

(c) Crédit photo Ilanit Illouz

53 Si la revue présente et organise des écritures, elle en organise aussi les activations. Elle présente ainsi lors de rendez-vous performatifs des activations, interprétations, réinterprétations des partitions par les artistes eux-mêmes ou par d'autres. Citons ainsi deux réinterprétations.

Lorraine Féline présentant une chorégraphie du geste 1 d'Édouard Boyer, initialement la description d'une posture corporelle nouvelle. La réinterprétation consistera, sur une musique de Sergio Leone, en la réalisation en direct et sur un rythme lent de la construction de la posture décrite.

\section{Figure 4}

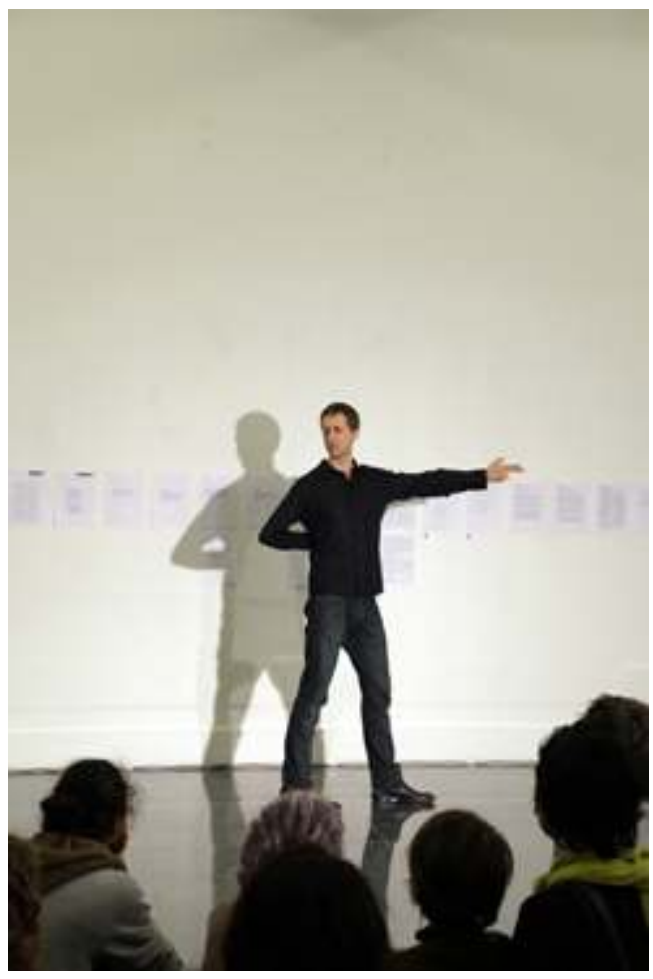

Édouard Boyer \& Lorraine Féline, Geste 1 (2011)

(c) Crédit photo Lucie Rocher 
À partir de la partition de Boris Charmatz, « la fonte », la danseuse Julia Cima proposait quant à elle, non pas de réaliser fidèlement la posture définie, mais de fondre collectivement. Une trentaine de personnes ont ainsi cédé à la gravité sur un temps commun et au fil de la partition projetée ${ }^{11}$.

Figure 5

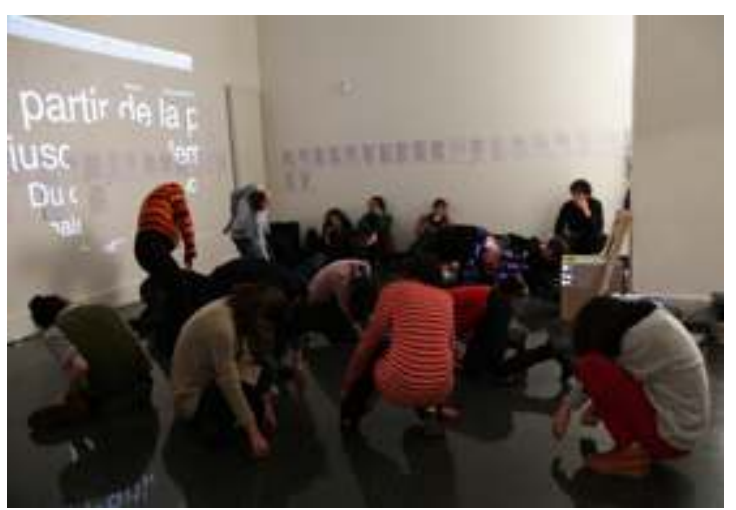

Julia Cima réactivant avec le public une partition de Boris Charmatz « La fonte »

(c) Crédit photo Lucie Rocher

Remarquons combien ces règles du jeu n'induisent ni durées, ni qualités, ni espaces mais suggèrent davantage pour leur interprète, un état, une posture, une combinaison initiale et laisse les modalités de réalisation libres. Elles défient ainsi l'idée que les "vertus» artistiques d'une œuvre seraient immanentes aux contours arrêtés de l'œuvre finie et close sur elle-même en introduisant une perspective nouvelle: dissocier le concept de son contexte d'exécution au point même de remettre en cause l'autorité du visible.

57 La figure de l'artiste démiurge et omnisciente s'ébrèche conjointement à ce nouveau statut de l'œuvre d'art.

L'existence de ses œuvres se fonde en quelque sorte sur un rituel qui orchestre l'apparition et la disparition à un instant précis et paradoxalement réactivable sans limites conditionnelles de temps ni d'espace. Ce rituel motive un rapport différent avec le spectateur, moins autoritaire voire parfois inversé lorsque ce dernier est laissé libre de concevoir et de réaliser l'œuvre elle-même.

59 À travers les exemples évoqués plus haut, de la Tool box de Lehmenn, ou les plugs de Thomasset, la démarche de l'artiste consiste moins à diriger des formes précises (composer) que de suggérer un préalable (initier). L'artiste se place désormais avant toute visibilité ou matérialité éventuelle de son œuvre. Sa posture serait celle d'un geste du préalable qui par définition est ce qui est dit, fait, examiné avant toutes choses. Son autorité n'est prégnante qu'en amont de l'œuvre, c'est-à-dire au seul moment de la conception dans la sphère du sensible et non dans le déroulement.

Le geste du préalable serait ainsi ce qui persiste de l'œuvre lorsque toute matérialité a disparu.

61 On est passé dès lors d'un ensemble de gestes à un geste unique, basculant du registre de l'existé à celui du préalable.

Un renversement du cerveau sur la main qui n'est pas sans faire résonner quelques échos à John Austin et sa performativité du quand dire c'est faire. 


\section{l'incarnation du geste technique, faisant de ce dernier non plus le média assujetti à une} technique mais bien la forme même.

64 loin d'avoir disparue, féconde des réalités aléatoires, infinies et anachroniques. Elles nous rappellent que tout est évolutif et que rien n'est vraiment stable. Ne serait-elle plus qu'un potentiel?

\section{NOTES}

1. Harald Szeeman, in Quand les attitudes deviennent formes, catalogue d'exposition Documenta 5, 1969.

2. G.W Hegel, Cours d'esthétique, t. I, Paris, Aubier, 1995, p. 105.

3. Extrait de l'article intitulé Art poétique élémentaire de Georges Amar, publié dans la Revue des ressources, mai 2004, Canada.

4. En cela je m'écarte de ce que prône Florence de Mérédieu qui voit dans la dématérialisation de l'art au cours du xxe siècle l'émergence de l'artiste « concepteur ", Histoire matérielle et immatérielle de l'art moderne, Paris, Larousse, 2004, (1994, Bordas pour la première édition), p. 554.

5. Selon Paul Virilio, dans l'entretien avec Fred Forest intitulé La fin des certitudes, paru dans le $n^{\circ}$ 122 de la revue Art press, février 1988, p. 16.

6.

http://www.entre-deux.org/projets-realises.php? subaction=showfull\&id=1229009425\&archive=\&start_from=\&ucat=5\& Évoquons une proposition similaire initié par Hans Ulrich Olbrist, DO IT commencée en 1993 est basée sur les modèles d'exposition ouverte et d'exposition en cours, en rassemblant des instructions écrites d'artistes postulant la multiplicité des interprétations, l'activité comme point de départ de ce qui est montré. http://www.e-flux.com/projects/do_it/homepage/do_it_home.html recense l'ensemble du catalogue.

7. http://www.stefanbruggemann.com

8. http://www.thomaslehmen.de/funktionen-277.html

9. http://www.vincent-thomasset.com/performances_09/091003_nuitb_home.html

10. Plusieurs activations ont eu lieu, l'une lors de la Nuit blanche 2009 dans la cour du musée Cognacq-Jay, à Paris.

11. Ces deux exemples d'activations ont eu lieu lors de l'exposition « Cuvre mode d'emploi », le 23 février 2011, à la galerie Michel Journiac / Laboratoire d'exposition de l'université Paris 1. 


\section{RÉSUMÉS}

À travers trois dispositifs de partitions (Tool Box, projet porté par Entre deux, la Tool Box du chorégraphe Thomas Lehmenn, revue Véhicule) il sera ici question de la partition considérée non plus comme notation de l'existant, ou à suivre, mais comme processus de création.

Véritable système de création, entre scénario et dispositif, les partitions artistiques et chorégraphiques donnent une nouvelle place à la structure, rendant visible ou objectivant un système préalable de création.

Le geste qui persiste de l'artiste n'est plus le fruit d'une action technique spécifique, mais bien la publicité de son intention préalable, symptôme d'une éthique de la "déproduction » pour bon nombre de pratiques artistiques contemporaines.

\section{INDEX}

Mots-clés : activation, art contemporain, chorégraphie, geste, partition, protocoles

\section{AUTEUR}

\section{MÉLANIE PERRIER}

Artiste chorégraphe, elle est parallèlement maitre de conférences en arts plastiques à l'université Paris 4 / IUFM de Paris. Chercheur au CERAP (Paris 1), elle est directrice du Laboratoire du Geste (rattaché à Art\&Flux de Paris 1), plateforme et équipe de recherche sur les esthétiques du geste en jeu dans le champ performatif et chorégraphique contemporain. Ses travaux actuels se concentrent sur l'élaboration d'outils critiques et d'analyse pour pratiques performatives, melanieperrier@laboratoiredugeste.com. 